\title{
Pathogenesis of some neurological immune diseases: ultrastructural and morphometrical observations on rat thymus
}

\author{
Daniela Cavallotti*, Vito D'Andrea ${ }^{\dagger}$, Francesco Saverio Pastore, \\ Francesca Maria Tranquilli Leali ${ }^{\ddagger}$ and Carlo Cavallotti ${ }^{\ddagger}$
}

\author{
Department of Neurosurgery University of Tor Vergata, Rome, Italy \\ *Operative Unit of Neurology, S. Spirito Hospital, Rome, Italy \\ ${ }^{\dagger}$ Department of Surgery and ${ }^{\ddagger}$ Department of Cardiovascular and Respiratory Sciences, University of Rome \\ "La Sapienza", Italy
}

Numerous studies on neuro-immuno-modulation indicate that the thymus is involved in many neurological diseases, including experimental allergic encephalomyelitis (EAE). Twenty Lewis rats were induced for EAE. At $X, X I I, X X$ and $X X X$ days post-inoculation the animals were killed, and the thymus was recovered and harvested. Specimens of thymus were submitted to morphological light microscopy analysis (1\% toluidine blue) and ultra-structural analysis (transmission electron microscopy). Significant morphometric data were collected by examining the images quantitatively and by statistically analysing the values. Our results show that the microenvironment of the thymus is severally involved in acute EAE. Thymocytes and reticular epithelial cells show many changes which are closely related to the pathogenesis of EAE. In particular we observed: (1) inside the cell an increase in intra-cytoplasmic vacuoles, and changes in the thickness of the nuclear membrane, mitochondria, rough endoplasmic reticulum, cellular inter-digitations and cellular electron-density; (2) outside the cell an increase in pericellular translucent halo, intercellular spaces, intercellular contacts and apoptotic and necrotic figures. The evidence of a thymic role in MS may suggest the intriguing therapeutic concept of thymectomy in the management of this neurological disease. [Neurol Res 2005; 27: $41-46]$

Keywords: Experimental allergic encephalomyelitis; multiple sclerosis; demyelinizing diseases; rat; thymus

\section{INTRODUCTION}

Experimental allergic encephalomyelitis (EAE) is a cellmediated autoimmune disease of the central nervous system (CNS) characterized by inflammatory infiltrates and demyelination of the nervous tissues ${ }^{1,2}$. Lewis rats are genetically predisposed for the induction of $\mathrm{EAE}^{3}$. This experimentally induced disease represents an animal model for the study of the human demyelinating disease multiple sclerosis (MS) ${ }^{4,5}$. Pathological changes of the thymus have been reported in cases of myasthenia gravis, MS and other neurological disorders ${ }^{6,7}$. In fact, the thymus plays an essential role in the stepwise process of differentiation and maturation of $T$ cell subsets. The most recent results reveal the presence in $\mathrm{EAE}$ and/or MS patients of several alterations in the number and function of immune organs. One hypothesis is that a segregation of T-suppressor lymphocytes in CNS may trigger a cell-mediated autoimmune reaction against

Correspondence and reprint requests to: Carlo Cavallotti, M.D., Section of Human Anatomy of the Department of Cardiovascular and Respiratory Sciences, University of Rome "La Sapienza" - Via A. Borelli, 5000161 Rome, Italy. [cavallotti@uniroma1.it] Accepted for publication February 2004. cellular elements of the same $\mathrm{CNS}^{8,9}$. Studies on the role of thymus in auto-immunity start around the years $1968-1969^{10,11}$. Recently it re-entered into fashion ${ }^{12-15}$ owing to the discovery of new antibodies which recognize particular antigens implied in these neurological diseases. In order to clarify the possible role of thymus microenvironment in the pathogenesis of $\mathrm{EAE}$, an ultra-structural analysis of thymus architecture in rats experimentally induced for EAE was made. Moreover, as new data, the quantitative analysis of images and the statistical analysis of data were performed.

\section{MATERIALS AND METHODS}

EAE was induced in adult Lewis rats by inoculation of an emulsion containing spinal cord tissue and Mycobacterium tuberculosis in complete Freund's adjuvant. The animals were treated according to the Convention of Helsinki on the utilization of animals in biomedical research. All procedures performed in this study were in accordance with the ethical standards of the responsible committee on animal experimentation and with the Declaration of Helsinki (1964) of the world Medical Association (amended in 1975 and 1983), 
published in Philosophy and Practice of Medical Ethics (British Medical Association, 1988). Most animals developed clinical signs of EAE within 12 days. Thymectomies were performed at X (pro-dromic symptoms), XII (early stage), XX (middle stage) and XXX (late stage) days post-inoculation (d.p.i.) of emulsion in rats and similarly in controls. Small pieces of the thymus were fixed in $2 \%$ glutharaldehyde for transmission electron microscopy analysis.

Male Lewis rats (220 g) and female Denkin Hartley guinea pigs (300 g) were obtained from Messrs Morini (S. Polo D'Enza, Italy) and kept under standard conditions, treated and stalled in authorized laboratories of our University. The control rats were injected with the vehicle alone (Freund's adjuvant in saline). EAE was induced, in male Lewis rats, by a single s.c. injection of $100 \mu \mathrm{g}$ in $500 \mu \mathrm{l}$ of an emulsion of guinea pig spinal cord into the hind foot pad. Local anaesthesia was performed with a saline solution of Novocain $0.5 \%$. The ratio of the contents of the emulsion was $100 \mathrm{mg}$ of spinal cord of guinea pig, homogenized in $4 \mathrm{ml}$ saline, emulsified with $1 \mathrm{ml}$ of complete Freund's adjuvant (CFA) (Difco, Detroit, MI) together with $10 \mathrm{mg}$ Mycobacterium tuberculosis (H37 RA Difco). The first clinical signs of EAE began day 12 post-immunization.

\section{Clinical assessment}

Rats were weighed and examined daily for the presence of neurological signs. Clinical signs: 1 , partial or complete loss of tail tonus; 2, paresis of the hind limbs; 3, complete paralysis of the hind limbs; 4, paralysis of the complete lower part of the body; 5, death due to EAE. Grades 2-4 are often accompanied by urinary and fecal incontinence ${ }^{16}$.

\section{Tissues sampling}

The rats were killed by decapitation at various times. The thymuses were rapidly removed and small specimens were used for each different technique.

\section{Usual laboratory staining}

The anatomical details of thymus specimens were analysed after staining with toluidine blue ( $1 \%$ solution) or hematoxylin and eosin.

\section{Transmission electron microscopy}

Thymus specimens were pre-fixed with $2.5 \%$ glutaraldehyde in $0.1 \mathrm{M}$ sodium cacodylate buffer. These prefixed tissue samples were post-fixed in $1 \%$ buffered osmium tetroxide for $1 \mathrm{~h}$, processed by conventional methods and embedded in epoxy resin Epon 812. Semithin sections were stained with $1 \%$ toluidine blue. Ultrathin sections were stained with uranyl acetate lead citrate and examined with a Philips CM 10 electron microscope.

\section{Quantitative analysis of images}

Quantitative analysis of the intensity of the staining samples was performed by means of a Quantimet
Analyser (Leica). The control values from samples incubated without dye were considered as "zero". Quantitative analysis of images (QAI) may provide incorrect results, because the main choices (i.e. the instructions for software) are made by each operator, according to his/her personal preferences. Therefore, it is mandatory to follow the rules carefully: the counts must be repeated at least three times using the doubleblind technique and should be performed by different people, on different analysers and with samples identified by a number or a letter. Another scientist, who identifies each sample and attributes specific values that then undergo statistical analysis, must obtain the final results. The values reported in our experiments represent the intensity of staining for each type of tissue and are expressed as Conventional Units (CU) \pm standard error of the mean; further details on QAI, including the definition of $\mathrm{CU}$, are reported in the manual of the Quantimet Leica $500^{17}$.

\section{Statistical analysis of data}

The statistical analysis performed included basic statistical methods such as mean values, maximum and minimum limits, variations, standard deviation (SD), standard error of mean (SEM) and correlation coefficients. Finally, by comparing the significant differences for each group with the corresponding values in the other homogeneous groups, a correlative analysis was obtained. Correlation coefficients denote a significant level when $p<0.001^{18}$.

\section{RESULTS}

Our results are shown in Figures $1-5$ and are tabulated in Tables 1 and 2. At 10 d.p.i. it is probable that no ultra-structural modification of the immunological reaction has a corresponding ultra-structural modification. However, large lymphocytes with nuclear and cytoplasmic characteristics resembling an activated population were present. The epithelial cells did not show any degenerative changes (Figure 1). The clinical and pathological signs of cerebral injury started at

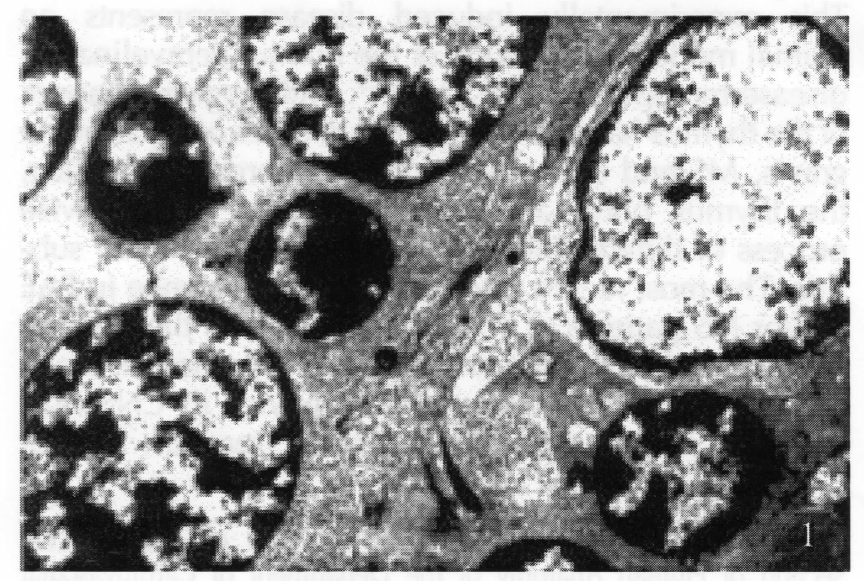

Figure 1: Transmission electron microscopy. Normal rat thymus. The normal morphology of reticular epithelial cells is shown. Magnification $\times 3000$ and reproduced here at $60 \%$ ) 
where the connections with epithelial cells were altered. These thymocytes had a nucleus with abundant chromatin and a nucleolus, and presented an increase of cytoplasm compartment (Figure 3).

At 30 d.p.i. epithelial cells had a more evident increase in intracytoplasmic vacuoles, and thymocytes showed some apoptotic figures. The epithelial cells showed an intense cytoplasmic vacuolisation with electron-lucent formations of different sizes and a massive dilatation of the nuclear membrane was evident. The thymocytes had variable irregular nuclei, some of these were apoptotic and surrounded by epithelial cells. The intercellular space was decreased and sometimes disappeared. A total thymic disorganization was evident into the cortical junctional, corticalmedullar and medullar areas.

The thymocytes were heterogeneous in shape and size. The small thymocytes were characterized by an extreme electron-dense nucleus, nuclear membrane alterations, scant cytoplasm and an irregular plasma membrane, surrounded by an electron-lucent zone (Figure 4). Only a few of intermediate size and some large thymocytes were present, with a nucleus composed of abundant chromatin and scant etero-chromatin and some mitochondria in the cytoplasm. Many necrotic figures and thymocytes with serious alterations of the nuclear membrane were visible. The intercellular space was filled with heterogeneous material of variable electron-density part of which resembled portions of cytoplasm with deleted plasma membranes. Also, epithelial cells had many degenerative changes: alterations of nuclear membrane, decrease of electrondense cytoplasm granules, increase of number of vacuoles and mitochondria, while the vessels were normal in size and shape (Figure 5). Table 1 summarizes our morphometric results on the percentage of cellular population and cortical-medullar ratio in rat thymus during the various phases of EAE. The major changes are: (1) decrease in medullar zone; (2) increase in cortical zone; (3) decrease in thymocytes; (4) increase in reticular epithelial cells and (5) increase in floating cells.

Table 2 deals with intra-cellular and extra-cellular changes in rat thymus during the various phases of the EAE. The major changes are in intercellular spaces, intercellular contacts, intercellular digitations, apoptotic figures, cellular electron density, mitochondria, cellular endoplasmic reticulum, cellular vacuoles and thickness of nuclear membrane.

\section{DISCUSSION}

Our results show intra-cellular and extra-cellular alterations in thymus of rats induced by EAE. The first symptoms and the first morphological alterations begin around XII d.p.i. and run with the disease's course. In MS and in experimental autoimmune diseases related to nervous system degeneration (such as EAE) the correlation between hystopathological features and clinical signs is often impossible to make. This is due to early degenerative processes and to the following regenerative events in CNS (de-myelinization and re-myelinization) ${ }^{19-21}$. The thymus gland is the major site in which Tlymphocytes undergo differentiation. Thymocytes of varying stages of differentiation are found in close contact with the epithelial cells, by the action of many adhesion molecules and their relative cell surface receptors $^{22-24}$. A close dialogue among the different

Table 1: Morphometric values of cellular elements of the thymus during the induction of EAE measured by QAI

\begin{tabular}{lccccc}
\hline Rat thymus & Normal & X days & XII days & XX days & XXX days \\
\hline Cortical zone (\%) & 62 & 68 & 74 & 84 & 92 \\
Medullar zone (\%) & 38 & 32 & 26 & 16 & 8 \\
No. of thymocytes $/ \mathrm{mm}^{2}$ & 50 & 42 & 31 & 21 & 12 \\
No. of reticular cells $/ \mathrm{mm}^{2}$ & 40 & 44 & 47 & 53 & 58 \\
No. of floating cells $/ \mathrm{mm}^{2}$ & 10 & 14 & 22 & 26 & 30 \\
\hline
\end{tabular}

Each value was obtained as CU (see Materials and methods) and was changed to a percentage according to the values reported in literature.

Table 2: Morphometric ultrastructural values of cellular constituents of the thymus during the induction of EAE measured by QAI

\begin{tabular}{|c|c|c|c|c|c|}
\hline Rat thymus & Normal & $\mathrm{X}$ days & XII days & $\mathrm{XX}$ days & $\mathrm{XXX}$ days \\
\hline Intercellular spaces & $3.2 \pm 0.6$ & $3.4 \pm 0.8$ & $6.3 \pm 1.2$ & $9.2 \pm 1.0$ & $12.1 \pm 0.8$ \\
\hline Apoptotic figures & $0.3 \pm 0.3$ & $0.5 \pm 0.3$ & $1.4 \pm 0.6$ & $3.6 \pm 1.6$ & $7.3 \pm 1.2$ \\
\hline Intercellular contacts & $3.6 \pm 1.3$ & $3.8 \pm 1.5$ & $3.9 \pm 1.2$ & $1.8 \pm 0.9$ & $0.8 \pm 0.4$ \\
\hline Electron density & $18.1 \pm 2.1$ & $19.3 \pm 2.4$ & $29.1 \pm 3.2$ & $34.5 \pm 2.9$ & $43.2 \pm 3.3$ \\
\hline Cellular inter-digitations & $9.6 \pm 1.4$ & $8.1 \pm 1.1$ & $6.4 \pm 0.9$ & $3.9 \pm 0.7$ & $1.8 \pm 0.6$ \\
\hline Rough endoplasm reticulum & $8.9 \pm 1.1$ & $11.4 \pm 1.3$ & $15.6 \pm 1.7$ & $19.3 \pm 1.6$ & $21.2 \pm 1.9$ \\
\hline Mitochondria & $16.4 \pm 1.8$ & $19.2 \pm 1.6$ & $22.4 \pm 2.1$ & $28.5 \pm 2.7$ & $33.3 \pm 3.2$ \\
\hline Cellular components & $5.1 \pm 1.2$ & $8.9 \pm 1.6$ & $9.3 \pm 2.1$ & $10.6 \pm 2.4$ & $14.4 \pm 1.8$ \\
\hline Intra-cytoplasmatic vacuoles & $2.6 \pm 0.6$ & $2.8 \pm 0.4$ & $2.8 \pm 0.5$ & $5.1 \pm 0.8$ & $7.3 \pm 1.1$ \\
\hline
\end{tabular}

Each value was expressed as CU \pm SEM (see Materials and methods). 
cellular components in the thymus is responsible for the thymic microenvironment required for thymocyte growth and differentiation ${ }^{25,26}$.

The interaction between thymocytes and the epithelial cells, plays a fundamental role in the development and maintenance of the immune response ${ }^{27}$. Furthermore, the role of the thymus in the pathogenesis of some neurological autoimmune disorders has been suggested and the demyelinization process seems to be related to the quality and quantity of infiltrated inflammatory components (macrophages and lymphocytes).

Changes in thymocyte subpopulations have been described during $\mathrm{MS}$ and $\mathrm{EAE}$ development ${ }^{28}$. The morphological modifications of the thymic gland corresponding to specific phases of the developing EAE were studied in our experiments. The clinical and pathological signs of cerebral injury started at 12 d.p.i. At the same time, ultra-structural findings show a depletion of thymocytes from the thymus and an increase of intercellular spaces due to a probable exit of lymphocytes from thymus into the blood, directed against the nervous tissue. The following ultra-structural features at 20 d.p.i. showed a thymic reorganization, together with a sporadic intrathymic death of thymocytes, related to a progressive alteration of epithelial cells. The death of thymocytes causes a progressive degeneration of thymus with an acme around 22 d.p.i. These observations suggest that thymic architecture needs to be well preserved in order to control the differentiation process of thymocytes, allowing a successful accomplishment of the disease.

The total thymus degeneration at 30 d.p.i. and the loss of tissue organization interfered on thymocytes development and their loss into the blood caused the progressive decrease of EAE and disappearance of clinical signs. Only $20 \%$ of rats survived while, on the contrary, about $80 \%$ of the treated animals died.

Progressive changes of thymic epithelial cells suggested the direct involvement of these cells in determining changes in thymus microenvironment and the development of EAE. The evidence of a thymic role in $\mathrm{EAE}$ and consequently also in MS may suggest the intriguing therapeutic concept of thymectomy in the management of these neurological disorders ${ }^{29,30}$.

In conclusion, we observed that, in control animals, the structure of the thymus epithelium which regulates T-cell subsets was normal, but that there were some alterations to the morphology of the thymus in EAE rats.

\section{ACKNOWLEDGEMENTS}

This study was supported by grants from University of Rome "La Sapienza" (Dip. 01.043.14). The authors are greatly indebted to Drs R. Steigherwalt and G. Palladini for their suggestions and criticism. Medline, the Internet and other informative consulting services of Dr ssa. Gaetana Cognetti (Biblioteca Istituto Regina Elena Roma) are gratefully acknowledged. The technical assistance of Mr D. Caporuscio, the photographic service of $\mathrm{Mr}$ G. Leoncini, the excellent secretarial work of Mrs S. Casamento are also gratefully acknowledged. The revision of the English language by Patrick Roger is also gratefully acknowledged.

\section{REFERENCES}

1 Ortiz-Ortiz L, Weigle W. Cellular events in the induction of experimental allergic encephalomyelitis in rats. J Exp Med 1976; 144: 604-616

2 Chow LH, Feurer C, Borel JF. Chronic relapsing experimental allergic encephalomyelitis in the Lewis rat: Studies on immune regulation. J Neuroimminol 1988; 19: 329-338

3 Goverman J, Brabb T. Rodent models of experimental allergic encephalomylitis applied to the study of multiple sclerosis. Lab Anim Sci 1996; 46: 482-488

4 Martin R. Immunological aspects of experimental allergic encephalomyelitis and multiple sclerosis and their application for new therapeutic strategies. J Neural Transmission 1997; 49: 53-67

5 Aknin SB, Morel RF. The role of the thymus in myasthenia gravis: Immuno-histological and immunological studies in 115 cases. Ann NY Acad Sci 1987; 50: 550-556

6 Martin R, Mcfarland H. Experimental immunotherapies for multiple sclerosis. Springer Semin Immunopathol 1996; 18: 1-8

7 Armstrong RM. Immunologic mechanism in neurologic diseases. Med Clin N Am 1972; 56: 515-527

8 Lynn Massman R, Edward AC, Sarka H, Ellsworth CA. Fluctuations of $\mathrm{T}$ - and B-cell subsets in basic protein induced experimental allergic encephalomyelitis in long tailed macaques. Clin Immunol Immunopathol 1987; 44: 93-101

9 Keywsky BA. Thymic nurse cells: Possible sites of T-cell selection. Immunol Today 1986; 16: 374-381

10 Metcalf D. The role of the thymus in auto-immunity. Bibl Haematol 1968; 29: 446-453

11 Denman AM. Anti-lymphocytic antibody and autoimmune disease: A review. Clin Exp Immunol 1969; 5: 217-249

12 Williams KC, Zhao W, Politopoulou G, et al. Inhibition of experimental allergic encephalomyelitis with an antibody that recognizes a novel antigen expressed on lymphocytes, endothelial cells, and microglia. Lab Invest 2000; 80: 313-326

13 Kaye JF, Kerlero de Rosbo N, Mendel I, et al. The central nervous system-specific myelin oligodendrocytic basic protein (MOBP) is encephalitogenic and a potential target antigen in multiple sclerosis (MS). J Neuroimmunol 2000; 102: 189-198

14 Weiner HL. The fine line between autoimmune and allergic encephalomyelitis. Nat Immunol 2001; 2: 193-194

$15 \mathrm{Liu} \mathrm{H}$, MacKenzie-Graham AJ, Kim S, et al. Mice resistant to experimental autoimmune encephalomyelitis have increased thymic expression of myelin basic protein and increased MBP specific T cell tolerance. J Neuroimmunol 2001; 115: 118-126

16 Matthaei I, Polman CH, De Groot CJA. Observer agreement in the assessment of clinical signs in experimental allergic encephalomyelitis. J Neuroimmunol 1989; 103: 215-222

17 Manuale dei Metodi Quantimet 500 Leica Microsystems Imaging Solutions, Clifton Road Cambridge, UK, 1997: pp. 1-102

18 Serio A. Appunti dalle lezioni di statistica sanitaria. Kappa, E. F. ed., Rome, 1997: pp. 1-186

19 Dal Canto MC, Wismiewski HN, Johnson AB, et al. Vesicular disruption of myelin in autoimmune demyelination. J Neurol Sci 1975; 24: 313-319

20 Lampert PW. Electron microscopic studies on ordinary and hyperacute experimental allergic encephalomyelitis. Acta Neuropathol 1967; 9: 99-106

21 Lampert PW. Demylination and remyelination in experimental allergic encephalomyelitis. J Neuropathol Exp Neurol 1965; 24: 371-377.

22 Couture C, Patel PC, Potworowski EF. A novel thymic epithelial adhesion molecule. Eur J Immunol 1990; 20: 276-284

23 Imhof BA, Ruiz P, Hesse B, Palacio R, Dunon D. EA-1 a novel adhesion molecule involved in the homing of progenitor $T$ lymphocytes to the thymus. J Cell Biol 1991; 95: 1069-1076

24 Singer KH, Denning SM, Whichard LP, et al. Thymocyte LFA-1 and thymic epithelial cell ICM-1 molecules mediate binding of activated human thymocytes to thymic epithelial cells. J Immunol 1990; 144: 2931-2933

25 Jonossy G, Thomas JA, Bollum FJ. The human thymic microenvironment: An immunohistologic study. J Immunol 1980; 134: 202208

26 King PD, Katz DR. Mechanism of dendritic cell function. Immunol Today 1990; 11: 206-210 
27 Ramson J, Fisher M, Mercer L, et al. Lymphokine mediated induction of antigen-presenting ability in thymic stromal cells. J Immunol 1987; 139: 2620-2625

28 Zaffaroni M, Caputo D, Ghezzia A, et al. Monoclonal antibody analysis of blood T-cell subsets in multiple sclerosis. Ital J Neurol Sci 1984; 5: 45-49
29 Rubin JW, Ellison RG, Moore HV, et al. Thymectomy in myasthenia gravis: The timing of surgery and significance of thymic pathology. Am Surg 1981; 47: 152-158

30 D'Andrea V, Biancari F, Cavallotti D, et al. Thymectomy and multiple sclerosis: Ultrastructural study of an experimental model. G Chir 1999; 20: 119-124 